\title{
INFLUÊNCIA DO MANEJO DO SOLO NA CONSERVAÇÃO, QUALIDADE SENSORIAL, TEOR DE NUTRIENTES E INCIDÊNCIA DE FITOPATIAS E FISIOPATIAS PÓS-COLHEITA DE PÊSSEGOS cv. CERRITO ${ }^{1}$
}

\author{
CARLOS ROBERTO MARTINS², RUFINO FERNANDO FLORES CANTILLANO ${ }^{3}$, \\ ROSA TREPTOW ${ }^{4}$, ROSELI DE MELLO FARIAS ${ }^{5}$, CESAR VALMOR ROMBALDI ${ }^{6}$
}

\begin{abstract}
RESUMO - Estudou-se o efeito do manejo do solo mantido com cobertura vegetal, na linha de plantio, na qualidade pós-colheita de pêssegos cv. Cerrito durante o armazenamento refrigerado. Os tratamentos constaram de frutas colhidas em pomares com solo com cobertura vegetal (aveia) e com cultivo tradicional (sem cobertura), em três estádios de maturação. O armazenamento foi feito em câmara fria a $0^{\circ} \mathrm{C}$ e umidade relativa do ar acima de $90 \%$. As avaliações da presença de fisiopatias e fitopatias, análise sensorial e análise de nutrientes foram feitas na colheita e após 6; 12 e 18 dias de armazenamento, mais três dias de simulação de comercialização. A análise sensorial demonstrou que as frutas colhidas em pomares com manejo do solo com cobertura vegetal apresentaram aparência, aroma, qualidade e sabor ao final do período de armazenamento superior às frutas de cultivo tradicional. Os atributos aceitação comercial e desidratação não apresentaram diferenças significativas. A coloração demonstrou ser superior em pêssegos provenientes de pomar com manejo do solo tradicional. A análise de nutrientes demonstrou maior conteúdo de N, Ca e B em frutas provenientes de pomar com manejo do solo com cobertura vegetal.
\end{abstract}

Termos para indexação: armazenamento refrigerado, cobertura vegetal, Prunus persica.

\section{SOIL MANAGEMENT EFFECTS IN RESPECT TO CONSERVATION AND SENSORIAL QUALITY, NUTRIENTS LEVELS AND INCIDENCE OF ROT AND PHYSIOLOGICAL DISORDERS OF PEACHES IN POST HARVEST}

\begin{abstract}
The effect of soil management, with mulching on the planting line on the quality of post harvest of peaches, cv. Cerrito, during cold storage was studied. The treatments were consisted by fruits harvested on orchards with mulching (oat) and without mulching (traditional cultivation) on three stages of maturation. The storage was performed at temperature $0^{\circ} \mathrm{C}$ and above $90 \%$ of air humidity. The presence of physiological disorders and rot evaluations, sensorial analysis and analysis of nutrients were performed at harvest and after 6, 12 and 18 days of storage, plus three days of commercialization simulation. The sensorial analysis showed that the fruits harvested on orchard management soil with mulching presented, on the final of storage period, a superior appearance, aroma, quality and flavor, than the others. The attributes commercial acceptance and dehydration didn't present significant differences. The coloration showed harder in coming peaches of orchard of management traditional soil. The analysis of nutrients showed larger content of $\mathrm{N}, \mathrm{Ca}$ and $\mathrm{B}$ in fruits harvest at orchard with management of soil with mulching.
\end{abstract}

Index terms: Cold Storage, Mulching, Prunus persica.

\section{INTRODUÇÃO}

Embora o consumo per capita de pêssegos no Brasil seja baixo, em torno de $0,25 \mathrm{~kg} / \mathrm{hab} / \mathrm{ano}$, comparado aos $5 \mathrm{~kg} / \mathrm{hab} / \mathrm{ano}$ em países como Itália, Espanha, França e Inglaterra (Madail \& Medeiros, 1998), houve crescimento da demanda desta fruta nos últimos anos. Em algumas regiões, como a de Porto Alegre, atingiu 1 kg/hab/ano em 2000 (Marodin \& Sartori, 2000). Aliado ao aumento da demanda, também cresceu o grau de exigência dos consumidores, o que tornou necessária uma nova postura do produtor para satisfazer o mercado.

A necessidade de validar sistemas de produção, que viabilizem a permanência do produtor no mercado, com lucratividade do pomar e a preservação do meio ambiente, justifica o estudo de novas estratégias.Torna-se necessária a evolução tecnológica que incorpore técnicas e práticas culturais mais produtivas, aliadas ao respeito ao ambiente e à obtenção de frutas de melhor qualidade (Martins, 2001).

A qualidade pós-colheita está condicionada à qualidade que as frutas atingem no pomar. Assim, o primeiro passo para assegurar a melhor qualidade de qualquer fruta para o consumidor é maximizar a qualidade do pomar. Atualmente, incentiva-se, dentro do sistema de produção integrada de frutas de caroço, a prática de cobertura vegetal do solo, para recuperar e evitar a

\footnotetext{
1 (Trabalho 206/2001). Recebido: 27/12/2001. Aceito para publicação: 04/06/2002. Trabalho desenvolvido com apoio Fapergs, CNPq, Embrapa e UFPel. Parte integrante da Dissertação Mestrado em Agronomia - área Fruticultura de Clima Temperado

2 Eng $^{\circ}$ Agr $^{\circ}$, Pós graduação em Agronomia, FAEM, UFPel, Pelotas, RS, e-mail marticar@ufpel.tche.br.

3 Eng $^{\circ}$.Agr ${ }^{\circ}$. D.Sc. Embrapa Clima Temperado, Pelotas, RS. E-mail: fcantill@cpact.Embrapa.br.

4 Msc. Economia Doméstica Embrapa Clima Temperado, Pelotas, RS.

5 Eng $^{\mathrm{a}} \mathrm{Agr}^{\mathrm{a}}$ mestranda Departamento Horticultura UFRGS E-mail roselifarias@bol.com.br

6 Eng $^{\mathrm{o}} \mathrm{Agr}^{\mathrm{o}}$ D.Sc. Prof. Departamento de Ciência e Tecnologia Agroindustrial/FAEM
} 
erosão, conservar a água, proteger o meio ambiente e melhorar a qualidade das frutas. Apesar da importância dos fatores de campo na frigoconservação, poucas pesquisas têm sido conduzidas para verificar a influência do manejo do solo na qualidade e no potencial de conservação de pêssegos.

Considerando o adequado manejo do solo como importante fator pré-colheita no sistema de produção de pêssegos, o presente trabalho teve por objetivo principal avaliar o efeito do manejo do solo na linha de plantio, na conservação e na qualidade pós-colheita de pêssegos cv. Cerrito, durante o período de armazenamento refrigerado.

\section{MATERIAL E MÉTODOS}

O trabalho foi conduzido na Embrapa Clima Temperado, utilizando pêssegos da cv. Cerrito, colhidos em pomar comercial de Pelotas, safra 1999/2000. Foram colhidas frutas provenientes de um pomar com dois sistemas: 1) sem cobertura vegetal na linha de plantio (cultivo convencional); 2) com cobertura vegetal de aveia-preta (Avena strigosa) na linha de plantio. A cobertura com aveia no pomar tem sido cultivada há três anos consecutivos. Em cada sistema de manejo, as frutas foram colhidas em três estádios de maturação, caracterizados pela coloração de fundo e de superfície, conforme Araújo (1998), sendo maduro incipiente (M2-75 \% coloração esverdeada e 25\% coloração final do fruto), meio-maduro (M3-50\% coloração final característica da cultivar) e maduro (M4-75\% da coloração final característica da cultivar). Para cada tratamento, foram armazenadas frutas nos três estádios de maturação à temperatura de $0^{\circ} \mathrm{C}$ e com U.R. de, aproximadamente, $90 \%$. As frutas foram mantidas por períodos de 6, 12 e 18 dias de armazenamento refrigerado. Em cada um destes períodos, também se realizou uma simulação de comercialização, mantendo-se as frutas durante três dias a $\pm 25^{\circ} \mathrm{C}$, após a qual foram realizadas as seguintes avaliações:

Análise de Nutrientes: Foi realizada análise de nitrogênio $(\mathrm{N})$, potássio $(\mathrm{K})$, cálcio $(\mathrm{Ca})$, magnésio $(\mathrm{Mg})$ e boro $(\mathrm{B})$, determinada segundo metodologia descrita por Freire (1998).

Análise Sensorial: Realizada com auxílio de uma equipe de julgadores, previamente treinados, pertencentes ao quadro da Embrapa Clima Temperado, segundo os métodos descritivos (ABNT, 1993). Os atributos avaliados contemplaram características externas e internas. Para avaliações dos aspectos externos, observaram-se: aparência, desidratação, coloração e aceitação comercial (padrão de comercialização das frutas). Para avaliações internas das frutas, avaliaram-se aroma e sabor mais a qualidade geral (avaliações internas e externas à fruta). A avaliação foi realizada por métodos descritivos, teste de avaliação de atributos, usando escalas não estruturadas de $9 \mathrm{~cm}$, cujo extremo esquerdo representou a menor nota, e o direito, a maior intensidade das características analisadas.

Fisiopatias e fitopatias: determinados por contagem de frutas com escurecimento da epiderme, lanosidade, desintegração e escurecimento interno e podridões.

O delineamento experimental foi completamente casualizado, com esquema fatorial, sendo a unidade experimental de 20 frutas, com três repetições. Os dados foram submetidos à análise da variância e a comparação de médias foi feita pelo teste DMS $(\mathrm{P} \leq 0,05)$.

\section{RESULTADOS E DISCUSSÃO}

Os dados apresentados na Tabela 1 indicam a influência do manejo do solo no teor de nutrientes da fruta. Pêssegos no estádio meio maduro (M3), cv. Cerrito, provenientes de pomar com cobertura vegetal, apresentaram 0,20 mg de nitrogênio /100g a mais do que os produzidos no cultivo tradicional. Estes mesmos resultados foram detectados com pêssegos cv. Chimarrita (Martins et al., 2001). Loreti \& Pisani (1986) relatam que o cultivo mínimo do solo, com a manutenção temporária ou permanente da cobertura verde, como alternativa ao cultivo sistemático do solo, traz como conseqüências a redução das perdas de nitrogênio por lixiviação, o aumento da quantidade de matéria orgânica, maior porosidade e elevação dos teores de nutrientes assimiláveis no solo, através da mineralização da biomassa, e, por conseqüência, frutas nutricionalmente mais equilibradas, com incremento na qualidade e na conservabilidade. Com a prática de cobertura verde, consegue-se reciclar grande parte dos nutrientes, e as aplicações de adubos são reduzidas (Marangoni, 2000).

Tanto o teor de potássio $(\mathrm{K})$ como de magnésio $(\mathrm{Mg})$ não foram influenciados pelo manejo do solo. O potássio é o nutriente que, de maneira geral, tem maior influência na coloração das frutas, já o magnésio influencia o teor de açúcar na polpa, sendo que o Ca e o Mg quase na total magnitude das frutíferas, também exercem influência sobre o aroma (Carvalho et al., 1994).

Em relação ao teor de cálcio $(\mathrm{Ca})$, mesmo não se constatando diferença significativa, houve uma pequena superioridade na quantidade deste nutriente nas frutas colhidas em pomar com manejo do solo com cobertura vegetal $(0,3 \mathrm{mg} \mathrm{Ca} / 100 \mathrm{~g})$. Embora não se tenha encontrado trabalhos na literatura com pêssegos, relacionando manejo do solo com equilíbrio nutricional e conservabilidade, em outras frutíferas, como maçãs, o armazenamento é afetado por vários fatores, como desequilíbrio de nutrientes no solo, principalmente a deficiência de Ca. Esta deficiência pode provocar o aparecimento de fisiopatias nas frutas, favorecendo a formação de podridões ou a manifestação de sintomas indesejáveis durante o processo de frigoconservação (Iuch, 2000).

Já quanto ao teor de Boro (B) dos pêssegos, houve influência dos sistemas de manejo do solo na sua concentração. Frutas provenientes de pomar com manejo do solo com cobertura vegetal apresentaram maior aporte deste nutriente, quando comparadas àquelas provenientes do pomar com manejo do solo tradicional. O B assume papel importante no funcionamento das membranas e da parede celular das frutas (Vendrell \& Carrasquer, 1994).

A cobertura vegetal constitui-se numa importante fonte nutritiva para o pomar. A biomassa produzida pode alcançar, através de decomposição sucessiva pela microflora, até 19 ton de peso fresco/ha, dependendo da espécie vegetal. Da mineralização desta biomassa, ficam disponíveis, respectivamente, $80 ; 13 ; 70 ; 13$ e $7 \mathrm{~kg} / \mathrm{ha}$ de N, P, K, Ca e Mg, os quais reciclam no solo e contribuem para balanço nutricional do pomar (Rombolá et al., 2000).

Em relação à análise sensorial (Tabela 2), pêssegos verdoengos (M2) produzidos em pomar com manejo do solo com cobertura vegetal apresentaram melhor aparência do que pêssegos produzidos no pomar com manejo do solo com cultivo tradicional. $\mathrm{O}$ atributo aparência decaiu na preferência dos 
julgadores com o aumento do período de armazenamento, independentemente da procedência das frutas. A aparência das frutas de caroço é representada pela uniformidade das características típicas de cada cultivar. Problemas como desuniformidade e defeitos aumentam com frutas em estádios avançados de maturação e períodos prolongados de armazenamento (Neri et al., 1996).

Os pessegueiros cultivados em pomar com manejo do solo com cultivo tradicional produzem frutas de melhor sabor em relação às produzidas em pomar com manejo do solo com cobertura vegetal. $\mathrm{O}$ fator que, provavelmente, influenciou positivamente no sabor, encontrado nos pêssegos produzidos em pomar com sistema tradicional, foi o maior teor de SST nas frutas provenientes deste sistema. O sabor melhora com o aumento do grau de maturação.

A maior permanência das frutas em condições de refrigeração proporciona sabor característico menos intenso. Aos 6 dias de armazenamento, os pêssegos não apresentaram diferença significativa no sabor, quanto à procedência das frutas. Ao expor as frutas a condições de ambiente, porém, o metabolismo da fruta retorna ao nível normal, desencadeando o consumo de açúcar e ácidos pelo aumento da respiração. Este processo refletese na diminuição do sabor característico do pêssego (Chitarra \& Chitarra, 1990; Crisosto et al., 1997).

Segundo Valero \& Altisent (1998), a relação entre açúcar e acidez (SST/ATT) é importante no equilíbrio do sabor dos pêssegos. Com o avanço da maturação, a acidez diminui e o teor de SST aumenta, sendo esta relação fortemente influenciada pelas condições climáticas, pela cultivar e pelo manejo do pomar.

O parâmetro aceitação comercial foi avaliado no sentido de o consumidor aceitar ou não os pêssegos dentro de um padrão de comercialização. A aceitação comercial diminui com o grau de maturação e período de armazenagem. As frutas provenientes de pomar com manejo do solo com cobertura vegetal apresentaram maior aceitabilidade comercial nos estádios meio-maduro e maduro. No estádio verdoengo, a maior aceitabilidade foi obtida com frutas produzidas em pomar com manejo do solo com cultivo tradicional.
Qualidade geral expressa o somatório das avaliações internas e externas à fruta. Deste modo com o aumento do grau de maturação, a qualidade geral foi incrementada. Somente pêssegos nos estádios meio-maduro e maduro, provenientes de pomar com manejo do solo com cobertura, foram significativamente superiores aos produzidos em pomar com manejo do solo com cultivo tradicional. Qualidade é determinada por propriedades físicas e químicas, controladas por constituintes químicos que são variáveis pelo metabolismo da fruta. Durante a maturação, acontecem trocas importantes nos constituintes físico-químicos e estes, por sua vez, alteram a qualidade sensorial. A qualidade geral diminui com o avanço do período de armazenamento. Somente aos 6 dias, as frutas provenientes de pomar com manejo do solo com cobertura vegetal apresentaram qualidade superior. Nos demais períodos de armazenamento, não houve diferença na qualidade geral quanto à procedência das frutas. Segundo López et al. (1998), o consumidor valoriza cada vez mais a qualidade organoléptica juntamente com a aparência externa, de forma que a preferência geral se dá por pêssegos suculentos e doces, com consistência dura e que tenham boa coloração tanto de polpa como de película.

Os pessegueiros cultivados em pomar com manejo do solo com cultivo tradicional produziram pêssegos apenas no estádio verdoengo de melhor coloração de que os de pomar com manejo do solo com cobertura vegetal. A coloração, assim como demais critérios de qualidade, depende essencialmente das características da cultivar, condições ambientais e de manejo (Byrne et al., 1991; Vendrell \& Carrasquer, 1994; Crisosto et al., 1997). Todos esses fatores têm uma contribuição relativa na intensidade da coloração e, por conseqüência, na qualidade e comportamento pós-colheita dos pêssegos.

Pêssegos verdoengos produzidos em pomar com manejo do solo com cultivo tradicional apresentaram coloração significativamente superior aos produzidos em pomar com manejo do solo com cobertura vegetal; entretanto, nos demais estádios de maturação, não houve diferença significativa.

Crisosto et al. (1997) descrevem que solos com teores elevados de $\mathrm{N}$ proporcionam reduções no avanço da maturação dos

TABELA 1 - Nutrientes em pêssegos meio maduros (M3), cv. Cerrito, colhidos em pomar com manejo do solo com cobertura vegetal com aveia e cultivo tradicional (sem aveia). Pelotas-RS, 2000.

\begin{tabular}{|c|c|c|c|c|c|}
\hline $\begin{array}{c}\text { Manejo do } \\
\text { Solo }\end{array}$ & $\mathrm{N}$ & $\begin{array}{c}\mathrm{K} \\
\mathrm{n}\end{array}$ & $\begin{array}{c}\mathrm{Ca} \\
0 \mathrm{~g}--\end{array}$ & $\mathrm{Mg}$ & $\begin{array}{c}\mathrm{B} \\
\mathrm{c}\end{array}$ \\
\hline Com aveia & $1,48 \mathrm{a}$ & $240 a$ & $4,1 \mathrm{a}$ & $11,5 \mathrm{a}$ & $2,7^{\mathrm{a}}$ \\
\hline Sem aveia & $1,28 \mathrm{~b}$ & $240 \mathrm{a}$ & $3,8 \mathrm{a}$ & $11,5 \mathrm{a}$ & $2,0^{\mathrm{a}}$ \\
\hline
\end{tabular}

Médias seguidas pela mesma letra não diferem entre si, pelo teste DMS (5\%).

TABELA 3 - Avaliação do número de pêssegos cv. Cerrito, com fisiopatias e fitopatias, colhidos em três estádios de maturação (verdoengo M-2; meio-maduro M-3 e maduro M-4) e durante o período de armazenamento, provenientes de pomares com manejo do solo com cobertura vegetal de aveia e com cultivo tradicional (sem aveia). Pelotas-RS, 2000.

\begin{tabular}{cccccccc}
\hline & \multicolumn{3}{c}{ Estádio de Maturação } & \multicolumn{3}{c}{ Armazenamento (dias) } \\
\cline { 3 - 8 } & & M-2 & M-3 & M-4 & 6 & 12 & 18 \\
\hline \multirow{2}{*}{ Fisiopatias } & Com aveia & 0 & 0 & 1 & 0 & 0 & 1 \\
& Sem aveia & 0 & 0 & 1 & 0 & 1 & 0 \\
& Com aveia & 0 & 0 & 1 & 0 & 0 & 1 \\
& Sem aveia & 0 & 1 & 2 & 1 & 0 & 2 \\
\hline
\end{tabular}


TABELA 2 - Avaliação Sensorial em pêssegos cv. Cerrito, colhidos em três estádios de maturação (verdoengo M-2; meio-maduro M3 e maduro M-4) e durante o período de armazenamento, provenientes de pomares com manejo do solo com cobertura vegetal de aveia e com cultivo tradicional (sem aveia). Pelotas-RS, 2000.

\begin{tabular}{|c|c|c|c|c|c|c|c|}
\hline & & \multicolumn{3}{|c|}{ Estádio de Maturação } & \multicolumn{3}{|c|}{ Armazenamento (dias) } \\
\hline & & M-2 & M-3 & M-4 & 6 & 12 & 18 \\
\hline \multirow{2}{*}{ Aparência } & Com aveia & $7,86 \mathrm{aA}$ & $6,23 \mathrm{aB}$ & $5,55 \mathrm{aC}$ & $7,12 \mathrm{aA}$ & $6,77 \mathrm{aB}$ & $5,75 \mathrm{aC}$ \\
\hline & Sem aveia & $7,40 \mathrm{bA}$ & $6,22 \mathrm{aB}$ & $5,4 \mathrm{aC}$ & $7,14 \mathrm{aA}$ & $6,00 \mathrm{bB}$ & $5,87 \mathrm{aC}$ \\
\hline \multirow[t]{2}{*}{ Sabor } & Com aveia & $2,37 \mathrm{bC}$ & $4,82 \mathrm{bB}$ & $6,58 \mathrm{aA}$ & $4,87 \mathrm{aA}$ & $4,65 \mathrm{bB}$ & $4,26 \mathrm{bC}$ \\
\hline & Sem aveia & $3,18 \mathrm{aC}$ & $5,17 \mathrm{aB}$ & $5,93 \mathrm{bA}$ & $4,79 \mathrm{aB}$ & $4,98 \mathrm{aA}$ & $4,51 \mathrm{aC}$ \\
\hline Aceitação & Com aveia & $4,52 \mathrm{bC}$ & $6,14 \mathrm{aA}$ & $5,20 \mathrm{aB}$ & $6,75 \mathrm{aA}$ & $5,78 \mathrm{aB}$ & $3,33 \mathrm{aC}$ \\
\hline Comercial & Sem aveia & $5,66 \mathrm{aA}$ & $5,47 \mathrm{bB}$ & $4,46 b C$ & $6,23 \mathrm{bA}$ & $5,89 \mathrm{aB}$ & $3,48 \mathrm{aC}$ \\
\hline Qualidade & Com aveia & $2,15 b C$ & $5,40 \mathrm{aA}$ & $7,07 \mathrm{aA}$ & $5,50 \mathrm{aA}$ & $4,87 \mathrm{aB}$ & $4,25 \mathrm{aC}$ \\
\hline Geral & Sem aveia & $3,09 \mathrm{aC}$ & $4,63 \mathrm{bB}$ & $6,40 \mathrm{bA}$ & $5,13 \mathrm{bA}$ & $4,72 \mathrm{aB}$ & $4,26 \mathrm{aC}$ \\
\hline \multirow[t]{2}{*}{ Coloração } & Com aveia & $1,06 \mathrm{bC}$ & $5,97 \mathrm{aB}$ & $7,91 \mathrm{aA}$ & $4,31 \mathrm{bC}$ & $5,46 \mathrm{bB}$ & $5,98 \mathrm{bA}$ \\
\hline & Sem aveia & $3,19 \mathrm{aC}$ & $6,16 \mathrm{aB}$ & $8,15 \mathrm{aA}$ & $4,81 \mathrm{aC}$ & $6,04 \mathrm{aB}$ & $6,65 \mathrm{aA}$ \\
\hline \multirow[t]{2}{*}{ Desidratação } & Com aveia & $0,98 \mathrm{aA}$ & $2,00 \mathrm{aC}$ & $2,58 \mathrm{aC}$ & $0,73 \mathrm{aA}$ & $1,40 \mathrm{bB}$ & $3,43 \mathrm{aC}$ \\
\hline & Sem aveia & $0,89 \mathrm{aA}$ & $1,84 \mathrm{aB}$ & $2,64 \mathrm{aC}$ & $0,52 \mathrm{aA}$ & $1,91 \mathrm{aB}$ & $2,94 \mathrm{bC}$ \\
\hline \multirow[t]{2}{*}{ Aroma } & Com aveia & $1,79 \mathrm{aC}$ & $5,66 \mathrm{aB}$ & $7,09 \mathrm{aA}$ & $5,29 \mathrm{aA}$ & $4,56 \mathrm{aB}$ & $4,69 \mathrm{aB}$ \\
\hline & Sem aveia & $1,41 \mathrm{bC}$ & $5,12 \mathrm{bB}$ & $6,49 \mathrm{bA}$ & $4,82 \mathrm{bA}$ & $4,15 \mathrm{bB}$ & $4,05 \mathrm{bB}$ \\
\hline
\end{tabular}

Médias seguidas da mesma letra, minúsculas nas colunas e maiúsculas nas linhas, não diferem entre si, pelo teste DMS (5\%).

pêssegos, induzindo a um pequeno desenvolvimento da coloração avermelhada e inibindo a mudança de coloração esverdeada para amarelada. Regiões de solo com teor de potássio (K) elevado têm proporcionado melhor coloração das frutas (Vendrell \& Carrasquer, 1994).

Não houve diferença significativa em relação à desidratação dos pêssegos quanto à procedência de frutas. A desidratação aumenta significativamente com o avanço do período de armazenagem e estádio de maturação, independentemente da procedência das frutas.

Pêssegos produzidos em pomar com manejo do solo com cobertura vegetal apresentaram aroma característico significativamente superior àqueles produzidos em pomar com manejo do solo com cultivo tradicional, independentemente do estádio de maturação e do período de armazenamento. A intensidade de aroma característico diminuiu significativamente com o período de armazenamento, no entanto, aumentou com o grau de maturação, independentemente da procedência das frutas.

Os compostos aromáticos também contribuem para a qualidade da fruta. $\mathrm{O}$ aroma dos pêssegos é determinado pela combinação ou superposição de elevado número de substâncias voláteis, como álcoois, acetonas, aldeídos, ésteres e outros compostos (Romojaro \& Riquelme, 1994). Atualmente, poucas informações são encontradas a este respeito. Trabalhos de pesquisas devem começar a ser desenvolvidos com ênfase neste tão importante atributo de qualidade.

Em relação às fisiopatias (distúrbios fisiológicos) e fitopatias (podridões), frutas com escurecimento da epiderme, lanosidade, desintegração da polpa, escurecimento interno e com podridões foram praticamente inexistente, não sendo constatadas diferen- ças significativas quanto ao sistema de manejo do solo, estádio de maturação e período de armazenamento (Tabela 3). Durante os 18 dias de armazenamento mais 3 dias de simulação de comercialização, tanto fisiopatias como fitopatias não se mostraram limitantes à conservação das frutas desta cultivar, independentemente do manejo do solo.

\section{CONCLUSÕES}

1. Pêssegos produzidos em pomares com manejo do solo com cobertura vegetal apresentam maior conteúdo de nitrogênio, cálcio e boro do que frutas colhidas em pomar com cultivo tradicional.

2. Pêssegos provenientes de pomares com manejo do solo com cobertura vegetal apresentaram aroma, aceitação comercial e qualidade geral ao final do período de armazenamento superior aos pêssegos provenientes de pomar com manejo do solo com cultivo tradicional.

3. Independentemente do manejo do solo, a qualidade pós-colheita dos pêssegos não foi influenciada por fisiopatias e fitopatias.

\section{REFERÊNCIAS BIBLIOGRÁFICAS}

ARAÚJO, P.J. Manejo e Conservação pós-colheita: fisiologia e tecnologia pós-colheitas do pêssego Instalação e manejo do pomar. In: MEDEIROS, C.A.B.; RASEIRA, M. do C. B.A cultura do pessegueiro. Brasília: Embrapa-SPI, 1998. p.318-339 
ASSOCIAÇÃO BRASILEIRA DE NORMAS TÉCNICAS. Métodos de análise sensorial de alimentos e bebidas. SP: ABNT NBR 12994, 1993.

BYRNE, D.; H. NIKOLIC; A. N. BURNS. E. E. Variability in sugars, acids, firmness, and color characteristics of peach genotypes. Journal American Society Horticultural Science, Alexandria v. 116, p. 1004-1006, 1991.

CARVALHO, J. G.; JUNIOR, J.P.O.; PAULA, M. B.; BOTREL, N. Influência dos nutrientes na qualidade de frutos. Informe Agropecuário, Belo Horizonte, v. 17, n 180, p. 52-55. 1994.

CHITARRA, M.I.F; CHITARRA, A. B. Pós-colheita de frutas e hortaliças: fisiologia e manuseio. Lavras: ESAL-FAEPE, 1990. p. 320.

CRISOSTO, C.H.; JOHNSON, R.S.; DEJONG, T. Orchard Factors Affecting Postharvest Stone Fruit Quality. HortSciense, Alexandria v. 32, n.5, p. 820-823. 1997.

FREIRE, C. J.S. Manual de métodos de análise de tecido vegetal, solo e calcário. Pelotas: Embrapa-CPACT, 1998. 208 p.

IUCH, V. L. Fatores pré-colheita que afetam a conservação. Boletim Informativo, Projeto de pesquisa em Horticultura para pequenos produtores no Sul do Brasil.

LÓPEZ, M.D.H.; MADRID, M.C.M.; BALLESTEROS, F.R.; MULA, M.S.; GARRIDO, D.V. Conservación frigorífica de Melocoton Parámetros de Calidad. Fruticultura Profesional, Espanha, n. 93, p. 55-59, 1998.

LORETI, F.; PISANI, P.L. Lavorazioni del terreno negli arboreti. Rivista di Agronomia, Itália, v. 20, n. 2-3, p.134-152, 1986.

MADAIL, J. C. M.; MEDEIROS, A.R.M. Economia da produção. In: MEDEIROS, C.A.B.; RASEIRA, M. do C. B. A cultura do pessegueiro. Brasília: Embrapa-SPI, 1998. p.318-339

MARANGONI, B. Esigence nutrizioli delle piante da frutto in clima temperato. In: ENCONTRO NACIONAL DE FRUTICULTURA, 3., 2000., Fraiburgo. Anais... Fraiburgo, SC, 2000. p. 5059.
MARODIN, G. A. B.; SARTORI, I.A. Situação das frutas de caroço no Brasil e no mundo. IN: SIMPÓSIO INTERNACIONAL DEFRUTAS DECAROÇO PÊSSEGOS, NECTARINAS E AMEIXAS, 2000, Porto Alegre. Anais... Porto Alegre, RS: Universidade Federal do Rio Grande do Sul - UFRGS, 2000. p. 7-16.

MARTINS, C.R. Qualidade Pós-Colheita de Pêssegos em Pomar cultivado com Aveia. 2001. 70 f. Dissertação (Mestrado em Fruticultura de Clima Temperado) - Faculdade de Agronomia Eliseu Maciel, Universidade Federal de Pelotas, Pelotas, 2001.

MARTINS, C.R; CANTILLANO, R.F.F; DELGADO, R.M.; TREPTOW, R.; ROMBALDI, C. Manejo do solo na conservação e na qualidade pós-colheita de pêssegos (Prunus persica (L) Batsch). Revista Brasileira de Fruticultura, Jaboticabal, v. 23, n.1,p. 55-58, 2001.

NERI, F.; VASSALLI, P.; BRIGATI, S. Valutazione organolettica di alcune cultivar di pesche e nettarine. Rivista di frutticoltura, Itália, n.7/8, p. 57-62, 1996.

ROMBOLÁ, A. D.; MORENO, T.; SCUDELLARI, D.; TAGLIAVINI, M.; MARANGONI, B. A nutrição das frutas de caroço na fruticultura eco-compatível. IN: SIMPÓSIO INTERNACIONAL DE FRUTAS DE CAROÇO PÊSSEGOS, NECTARINAS E AMEIXAS, 2000, Porto Alegre. Anais... Porto Alegre, RS: Universidade Federal do Rio Grande do Sul - UFRGS, 2000. p. 41-60.

ROMOJARO, F.; RIQUELME, F. Critérios de calidad del fruto. Cambios durante la maduración. Identificación de criterios no destructivos. In: VENDRELL, M.; AUDERGON, J.M.Calidad postcosecha y productos derivados en frutos de hueso. Lleida, 1994. p. 55-79.

VALERO, C.; ALTISENT, M.R. Equipos de medida de calidad organoléptica en frutas. Fruticultura Profesional, Espanha, n. 95, p. 38-45, 1998.

VENDRELL, M.; CARRASQUER, A. M. Fisiologia postcosecha de frutos de hueso. In: VENDRELL, M.; AUDERGON, J.M. Calidad post-cosecha y productos derivados en frutos de hueso. Lleida, 1994. p. 37-55. 